\title{
IMPROVEMENT OF HOT-CARRIER RESISTANCE AND RADIATION HARDNESS OF $n$ MOSFETS BY IRRADIATION-THEN-ANNEAL TREATMENTS
}

\author{
Kuei-Shu Chang-Liao and Jenn-Gwo Hwu \\ Room 414, Department of Electrical Engineering, National Taiwan University, Taipei, Taiwan, \\ Republic of China
}

(Received 10 October 1990; in revised form 26 December 1990)

\begin{abstract}
The improvement of hot-carrier resistance and radiation hardness in $n$-channel MOSFETs by a novel technique is studied. This technique includes a Co-60 (1 M rad) irradiation and a subsequent anneal in $\mathrm{N}_{2}$ at $400^{\circ} \mathrm{C}$ for $10 \mathrm{~min}$. The hot-carrier-induced instability and the radiation-induced degradation in MOSFETs are examined from the shifts of threshold voltage, transconductance, and drain current. It is observed that the sample after irradiation-then-anneal treatment shows more resistance to hot carrier and radiation damage than that without treatment. The effects are explained by a model involving strain relaxation induced near the $\mathrm{SiO}_{2} / \mathrm{Si}$ interface by irradiation.
\end{abstract}

\section{INTRODUCTION}

The hot-carrier-induced device degradation is important to the long-term reliability of MOSFETs. The degradation is due to the increase of the internal electrical field as a result of device scaling or from the use of high power supply voltage. Several approaches have been reported which reduce the degradation[1-4]. Some make changes in device structures, and others add additional process steps during device fabrication. Degradation due to radiation damage is also important to MOSFETs. There are strong needs for radiation-hardened MOSFETs in space-satellite and nuclear-plant applications. Several approaches to improve the radiation hardness have been investigated in recent years[5-7]. Generally, workers have employed variations in fabrication [5,6] or designed new structures[7].

Recently, we have found that both the hotelectron-induced degradation and the radiationinduced degradation in MOS capacitor devices can be dramatically improved by repeated irradiation-thenanneal (ITA) treatments [8,9]. These improvements can be explained by a bond strain gradient model[10]. Briefly, it is known that the larger the $\mathrm{SiO}_{2} / \mathrm{Si}$ interface strain, the worse the hot carrier and radiation hardness of the oxide insulator $[3,8,9]$. We believe that irradiation may release the interface strain and that the radiation-induced damage can be suitably annealed out $[8,9]$. Therefore the ITA treated MOS capacitors should become more hot-carrier and radiation-hard than those without ITA treatment. Since the ITA treatment needs no change in the device fabrication process, it should be useful in practice.

In this work, the ITA treatment was applied to $n$-channel MOSFETs for reducing hot-carrier and radiation-induced degradation. Interestingly, it is found that the ITA-treated MOSFET devices yield smaller shifts in threshold voltage, transconductance, and drain current during hot-carrier stressing than the control devices. Also, it is found that the radiation-induced change of the drain current in the ITA-treated MOSFET is smaller than that for a control device. Therefore, the ITA treatment is useful for MOSFETs in the improvement of hot-carrier resistance and radiation hardness.

\section{EXPERIMENTAL}

The n-channel MOSFETs (channel length $L=30 \mu \mathrm{m}$, channel width $W=400 \mu \mathrm{m})$ used in this study were fabricated on (100)-oriented $p$-type silicon wafers with a resistivity of $4-10 \Omega \mathrm{cm}$. It is noted that the long-channel MOSFETs discussed in this work are important to industry application since they are still prone to hot-electron-induced degradation in analog circuits where a $10 \mathrm{~V}$ power supply is employed[11]. After standard cleaning, wet oxides of $7000 \AA$ were formed at $1000^{\circ} \mathrm{C}$ for $180 \mathrm{~min}$. The source/drain regions were then defined by photolithography. The source and drain were formed by ion implantation $\left(5 \times 10^{15} / \mathrm{cm}^{2}, 100 \mathrm{keV}\right)$ and were then annealed at $900^{\circ} \mathrm{C}$ for $30 \mathrm{~min}$ to activate the implants. After the oxide of the active regions was removed, $320 \AA$ gate oxide was grown at $950^{\circ} \mathrm{C}$ for $20 \mathrm{~min}$. Finally, after $\mathrm{Al}(1 \% \mathrm{Si})$ metallization and patterning, the devices were postmetallization annealed in $\mathrm{N}_{2}$ at $350^{\circ} \mathrm{C}$ for $10 \mathrm{~min}$.

The ITA treatment was performed after sample preparation. This ITA treatment was performed by a Co-60 irradiation with a total dose of $10^{6} \mathrm{rad}$ $\left(\mathrm{SiO}_{2}\right)$ then an anneal in $\mathrm{N}_{2}$ at $400^{\circ} \mathrm{C}$ for $10 \mathrm{~min}$. Note that the MOSFET devices were not biased during 
irradiation and the dose rate of irradiation was about $100 \mathrm{rads}\left(\mathrm{SiO}_{2}\right) / \mathrm{s}$ in this work. The MOSFETs with and without (control) ITA treatment were compared. The channel-hot-carrier (CHC) stressing experiments were performed by using an Hp4145 semiconductor parameter analyzer[12]. The stressing condition was decided according to the well known bell-shaped curve of substrate current $I_{\mathrm{SUB}}$ as a function of gate voltage $V_{\mathrm{G}}$ under a certain fixed drain voltage $V_{\mathrm{D}}$. Figure 1 shows the $I_{\text {SUB }}$ vs $V_{\mathrm{G}}$ curve of an $n$ MOSFET at $V_{\mathrm{D}}=15 \mathrm{~V}$. A $V_{\mathrm{G}}$ of $5.8 \mathrm{~V}$ was chosen according to the maximum value of substrate current shown in Fig. 1. In this work, the $\mathrm{CHC}$ stressing experiments were performed at $V_{\mathrm{D}}=15 \mathrm{~V}$ and $V_{\mathrm{G}}=5.8 \mathrm{~V}$. The source voltage $V_{\mathrm{S}}$ and the substrate bias were set to $0 \mathrm{~V}$. Note that since both of the $I_{\mathrm{SUB}}$ and the maximum channel electric field $E_{\mathrm{MAX}}$ are important to the characterization of hot-carrier-induced device degradation in long channel MOSFETs[11,13], they should be of the same condition for each stress experiment. The threshold voltage shift $\Delta V_{\mathrm{TH}}$, the percentage degradation of transconductance $\Delta G_{\mathrm{M}} / G_{\mathrm{M} 0}$, and the percentage degradation of drain current $\Delta I_{\mathrm{D}} / I_{\mathrm{D} 0}$ were recorded during stressing. In this work, the data of measurements and characterization were acquired after $0,10,30,70$ and $150 \mathrm{~min}$ of stressing.

To investigate the radiation-induced device degradation, the device parameters are examined before and after Co-60 (1 M rads) irradiation. The values of $\Delta V_{\mathrm{TH}}, \Delta G_{\mathrm{M}} / G_{\mathrm{M} 0}$, and $\Delta I_{\mathrm{D}} / I_{\mathrm{D} 0}$ are compared between the ITA treated and the control (without ITA) samples. It is known that the smaller the device parameter shift, the better the radiation hardness. Note that the threshold voltage $V_{\mathrm{TH}}$ is obtained from the extrapolated value of $I_{\mathrm{D}}$ vs $V_{\mathrm{G}}$ curve at $V_{\mathrm{D}}=0.1 \mathrm{~V}$. The transconductance $G_{\mathrm{M}}$ is the maximum slope of $I_{\mathrm{D}}$ vs $V_{\mathrm{G}}$ curve at $V_{\mathrm{D}}=0.1 \mathrm{~V}$. For $I_{\mathrm{D}}$

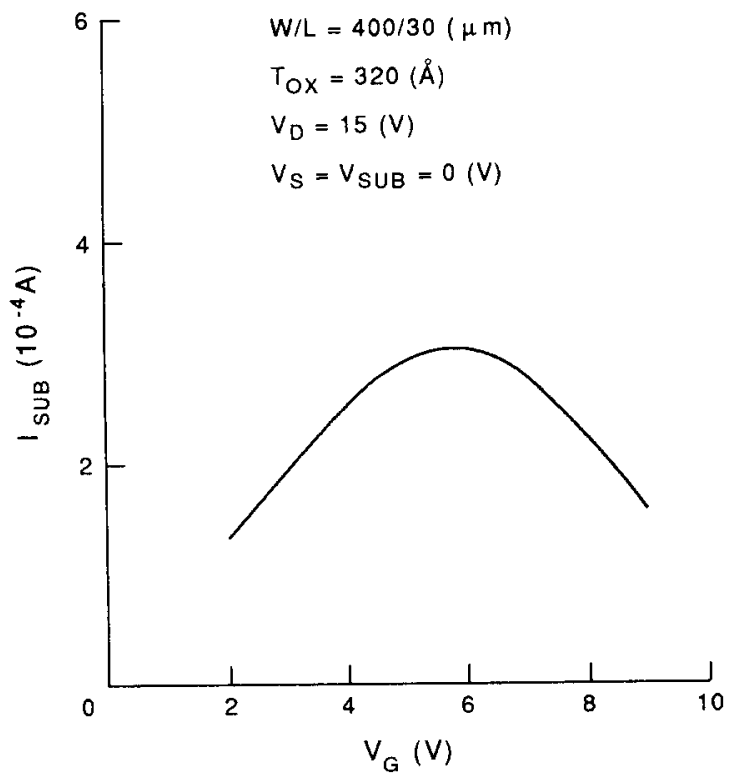

Fig. 1. $I_{\mathrm{SUB}}$ vs $V_{\mathrm{G}}$ at $V_{\mathrm{D}}=15 \mathrm{~V}$.

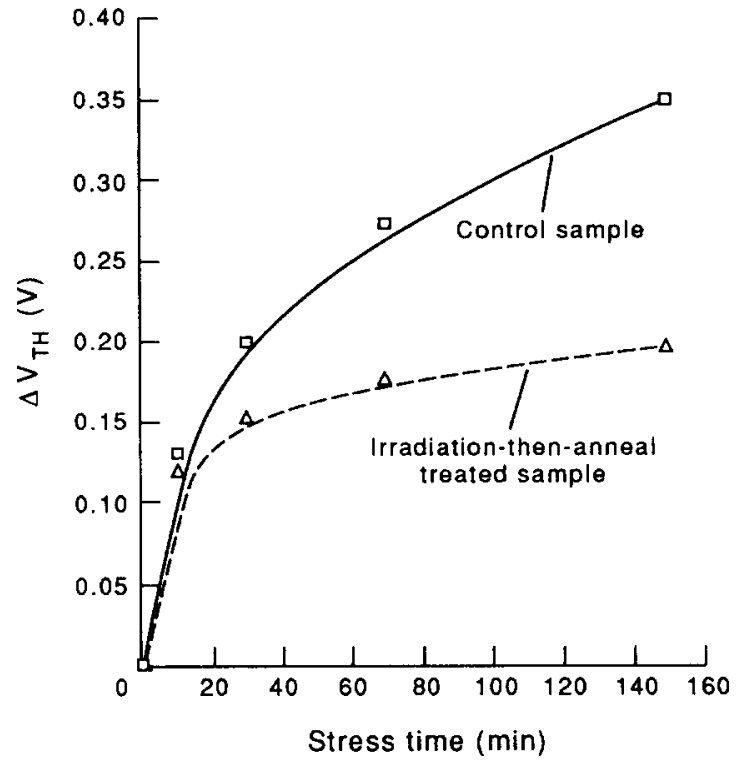

Fig. 2. Stress time dependence of threshold voltage shift $\Delta V_{\mathrm{TH}}$.

measurements, two conditions are compared: (1) linear region, $V_{\mathrm{D}}=0.1 \mathrm{~V}, V_{\mathrm{G}}=5 \mathrm{~V}$; and (2) saturation region, $V_{\mathrm{D}}=5 \mathrm{~V}, V_{\mathrm{G}}=5 \mathrm{~V}$.

\section{RESULTS AND DISCUSSION}

Figure 2 shows the $\Delta V_{\mathrm{TH}}$ as a function of $\mathrm{CHC}$ stressing time for a control and an ITA treated MOSFETs. It is observed that the magnitude of $\Delta V_{\mathrm{TH}}$ is substantially reduced for the ITA treated sample. Similarly, the $\Delta G_{M} / G_{M 0}$ as a function of CHC stressing time was also examined and was shown in Fig. 3. The magnitude of $\Delta G_{\mathrm{M}} / G_{\mathrm{M} 0}$ is also reduced for the

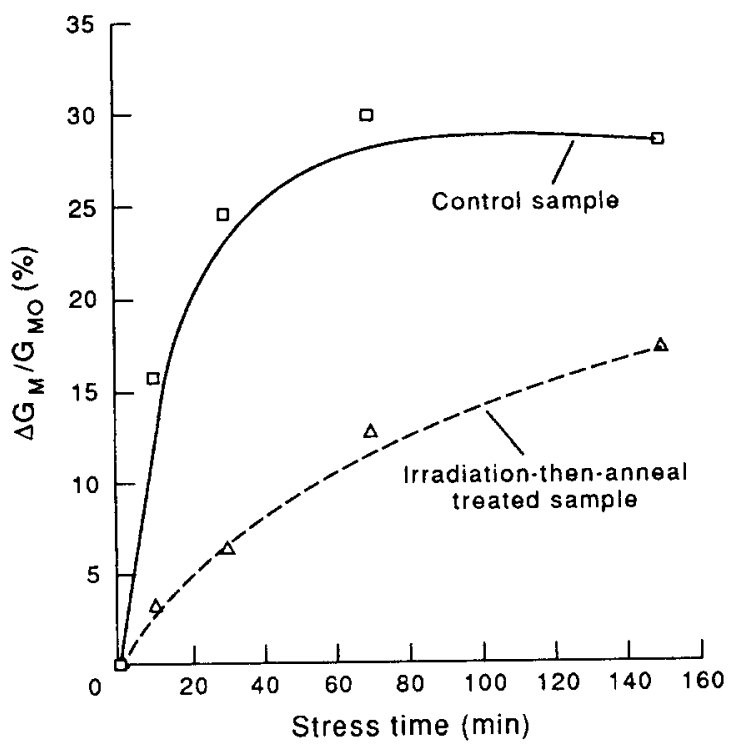

Fig. 3. Stress time dependence of transconductance percentage degradation $\Delta G_{\mathrm{M}} / G_{\mathrm{M} 0}$. 
ITA treated sample. It is clear that the ITA-treated MOSFET is more resistant to the hot-carrier induced degradation than the control device. It is known that both $\Delta V_{\mathrm{TH}}$ and $\Delta G_{\mathrm{M}}$ account for the hot-carrier induced interface trap generation[14]. Therefore, the results in both Figs 2 and 3 show that the generation of interface traps is suppressed in ITA treated oxides. Figure 4 shows the dependence of $\Delta I_{\mathrm{D}} / I_{\mathrm{D} 0}$ in the linear region on the hot-carrier stressing time. It also clearly shows that the ITA-treated $n$ MOSFET is more resistant to hot-carrier induced degradation than the control device. Similarly, the dependence of $\Delta I_{\mathrm{D}} / I_{\mathrm{D} 0}$ in the saturation region on the hot-carrier stressing time is also examined, as shown in Fig. 5. The improvement is quite clear for the ITA treated sample. It is known that $I_{\mathrm{D}}$ depends on $V_{\mathrm{TH}}$ and $G_{\mathrm{M}}$. Since $\Delta I_{\mathrm{D}}$ is reduced, it is believed that $\Delta V_{\mathrm{TH}}$ or $\Delta G_{\mathrm{M}}$ must also be reduced. This is consistent with the fact that the ITA treatment can reduce the generation of interface traps induced by hot-carrier stressing as described above. Since the stability of $I_{\mathrm{D}}$ is one of the most important electronic characteristics of MOSFET device performance, the ITA treatment is a useful method to improve the hot carrier resistance of MOS-based integrated circuit (I C).

Figure 6(a) shows the $I_{\mathrm{D}}$ vs $V_{\mathrm{D}}$ curves for a control sample before and after Co-60 (1 M rads) irradiation. As can be seen from this figure, the radiation damage is serious in the control sample since the $I_{\mathrm{D}}$ is decreased significantly after irradiation. The value of $\Delta V_{\mathrm{TH}}, \Delta G_{\mathrm{M}} / G_{\mathrm{M} 0}$, and $\Delta I_{\mathrm{D}} / I_{\mathrm{D} 0}$ (linear region) for this sample due to irradiation are $0.63 \mathrm{~V},-24 \%$ and $-27 \%$, respectively. Figure $6(\mathrm{~b})$ shows the $I_{\mathrm{D}}$ vs $V_{D}$ curves for an ITA treated sample before and after irradiation. Obviously, the radiation-induced decrease in $I_{\mathrm{D}}$ is reduced. The values of $\Delta V_{\mathrm{TH}}$,

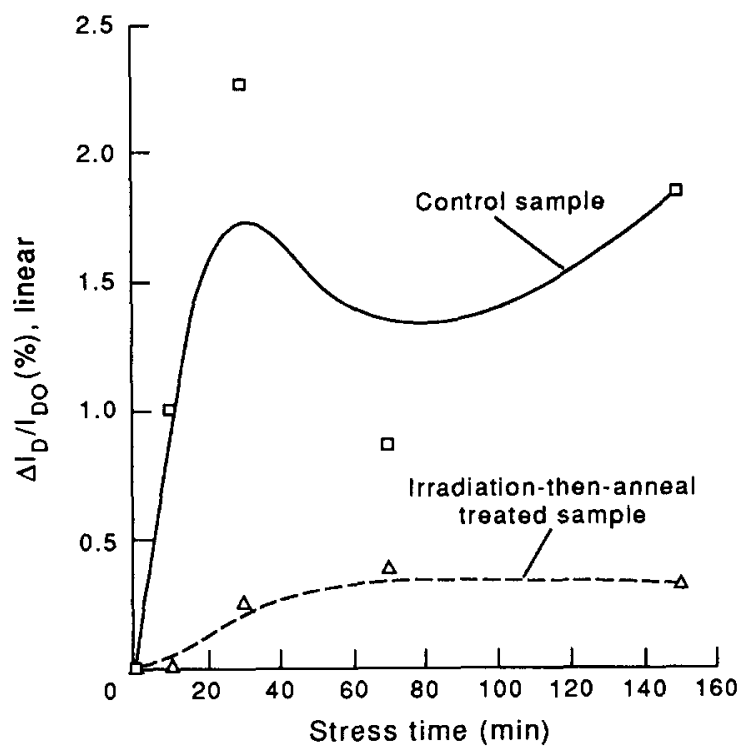

Fig. 4. Stress time dependence of drain current percentage degradation $\Delta I_{\mathrm{D}} / I_{\mathrm{D} 0}$ in linear region.

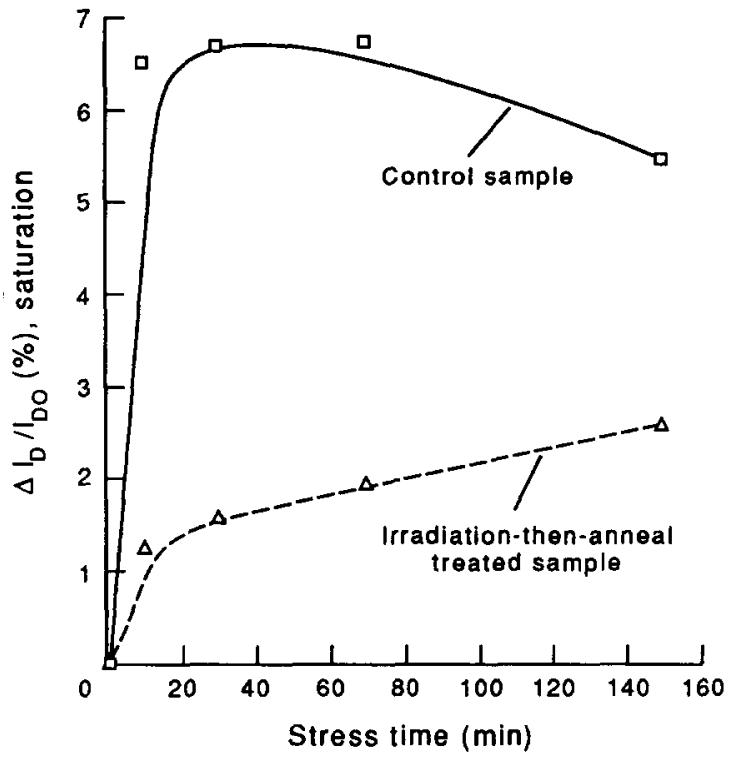

Fig. 5. Stress time dependence of drain current percentage degradation $\Delta I_{\mathrm{D}} / I_{\mathrm{D} 0}$ in saturation region.

$\Delta G_{\mathrm{M}} / G_{\mathrm{M} 0}$, and $\Delta I_{\mathrm{D}} / I_{\mathrm{D} 0}$ (linear region) for this sample due to irradiation are $0.2 \mathrm{~V},-19 \%$ and $-10 \%$, respectively. They are much smaller than those shown in Fig. 6(a). Thus it is evident that the radiation hardness can be significantly improved by the ITA treatment. It is noted that the $I_{\mathrm{D}}-V_{\mathrm{D}}$ curves before irradiation in Fig. 6(a) and (b) are not completely identical. The $V_{T H} s$ for the control and the ITA treated samples before irradiation are $-1.35 \mathrm{~V}$ and $-1.14 \mathrm{~V}$, respectively. There is little difference among them. This is mainly due to the fact that the anneal treatment used in this work does not completely remove the radiation-induced damage in $n$ MOSFETs. Further study of the choices of the total dose and anneal temperature used in the ITA treatment is worthwhile. However, from the results observed above, we can at least conclude that the ITA treatment is an efficient method to reduce the hot-carrier and radiation-induced degradation in $n$ MOSFETs.

It is hypothesized that the improved hot-carrier resistance and radiation hardness are due to the relaxation of the $\mathrm{Si} / \mathrm{SiO}_{2}$ interface strain after ITA treatment. According to the bond-strain gradient model[10], a reduced bond strain gradient leads to an increased oxide hardness. The ionizing radiation breaks a strained bond leaving an $\mathrm{Si}^{+}$site and a nonbridging oxygen center that could migrate toward the $\mathrm{SiO}_{2} / \mathrm{Si}$ interface to form interface traps. Therefore, we believe that radiation releases the interface strain, although it introduces interface traps and positive charges near the $\mathrm{SiO}_{2} / \mathrm{Si}$ interface. Since most of the radiation-induced positive charge and interface traps can be annealed out[15], the $\mathrm{SiO}_{2} / \mathrm{Si}$ interface strain will suitably be reduced by ITA treatment. This therefore gives an improved interfacial property for MOSFETs. 
(a)

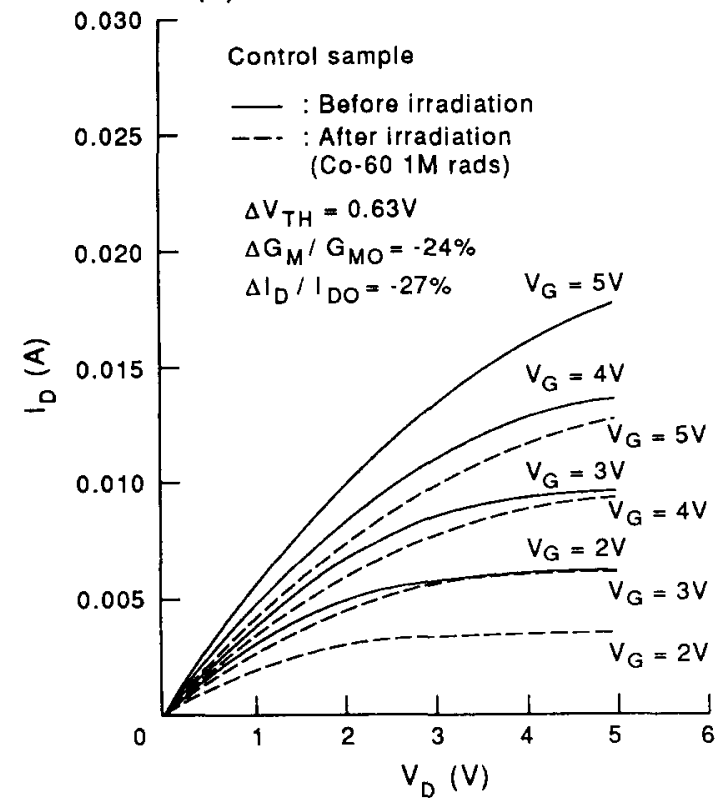

(b)

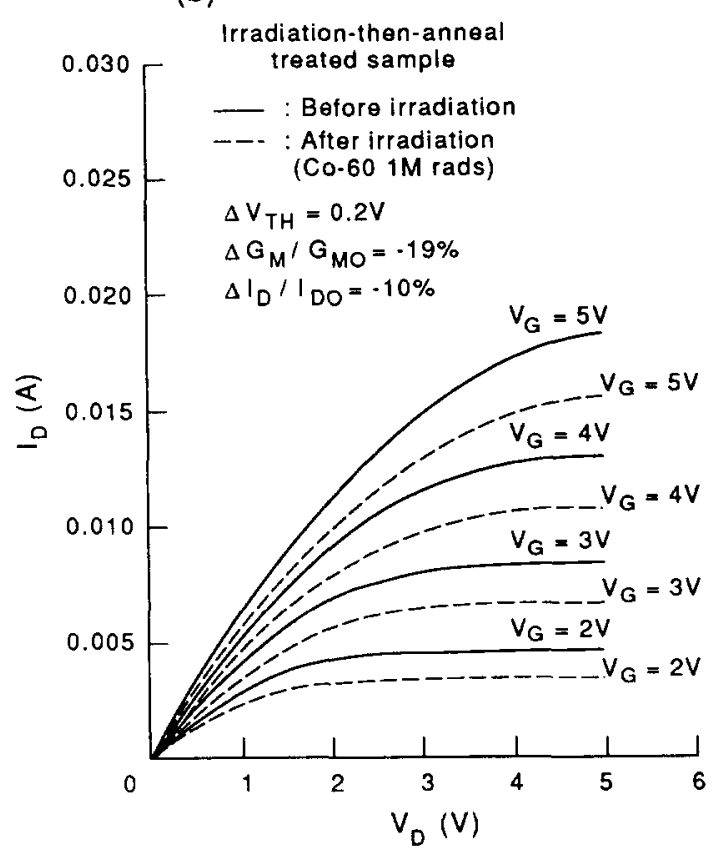

Fig. 6. $I_{\mathrm{D}}$ vs $V_{\mathrm{D}}$ characteristics before and after Co-60 (1 M rads) irradiation for (a) the control sample and (b) the ITA treated sample.

\section{CONCLUSIONS}

It is shown that the hot-carrier resistance and radiation hardness are much improved by ITA treatment. The improvements have been explained by the $\mathrm{SiO}_{2} / \mathrm{Si}$ interface strain relaxation. Since this treatment is performed after sample preparation, it is suitable for most of the MOSFET applications. With the present trend of device size scaling down, it is worthwhile to apply this method to submicron channel length devices.

Acknowledgement-The authors want to thank the National Science Council of the Republic of China for supporting this work under contract no. NSC80-0404-E002-01.

\section{REFERENCES}

I. S. Ogura, P. J. Tsang, W. W. Walker, D. L. Critchiow and J. F. Shepard, IEEE Trans. Electron Devices ED-27, 1359 (1980).

2. Y. Nishioka, K. Ohyu, Y. Ohji, N. Natuaki, K. Mukai and T. P. Ma, IEEE Electron Device Lett. EDL-10, 141 (1989).
3. K. P. MacWilliams, L. F. Halle and T. C. Zietlow, IEEE Electron Device Lett. EDL-11, 3 (1990).

4. K. K. Ng, C. S. Pai, W. M. Mansfield and G. A. Clarke, IEEE Electron Device Lett. EDL-11, 45 (1990).

5. G. B. Roper and R. Lowis, IEEE Trans. Nucl. Sci. NS-30, 4110 (1983).

6. Y. Wang, Y. Nishioka, T. P. Ma and R. C. Barker, IEEE IRPS, p. 145 (1988).

7. H. Hatano, I. Yoshii, M. Shibuya, S. Takatuka, T. Shinohara, T. Noguchi, K. Yamamoto, H. Fuji and R. Abe, IEEE Trans. Nucl. Sci. NS-36, 2435 (1989).

8. J. G. Hwu and J. T. Chen, IEEE Electron Device Lett. EDL-11, 82 (1990).

9. J. G. Hwu and S. L. Fu, Solid-St. Electron. 32, 615(1989).

10. F. J. Grunthaner, P. J. Grunthaner and J. Maserjian, IEEE Trans. Nucl. Sci. NS-29, 1462 (1982).

11. T. Y. Chan, C. L. Chiang and H. Gaw, IEDM Tech. Dig., p. 196 (1988).

12. I. C. Chen, J. Y. Choi, T. Y. Chan and C. Hu, IEEE Trans. Electron Devices ED-35, 2253 (1988).

13. C. Hu, S. C. Tam, F. C. Hsu, P. K. Ko, T. Y. Chan and K. W. Terrill, IEEE Trans. Electron Devices ED-32, 375 (1985).

14. J. Y. Choi, P. Keung, C. Hu and W. F. Scott, J. Appl. Phys. 65, 354 (1989).

15. E. H. Nicollian and J. R. Brews, MOS (Metal Oxide Semiconductor) Physics and Technology, p. 798. WileyInterscience, New York (1982). 